\title{
Velhice e sexualidade na pós-modernidade: um estudo sobre o corpo e o prazer
}

\author{
Old age and sexuality in post-modernity: a study on body and pleasure \\ Vejez y sexualidad en la posmodernidad: un estudio sobre el cuerpo y el placer
}

Richardson Lemos de Oliveira ORCID: https://orcid.org/0000-0002-4523-2337 Universidade do Estado do Rio de Janeiro, Brasil

E-mail: richardsonmedicina@gmail.com

Bruno Eduardo de Menezes Pequeno ORCID: https://orcid.org/0000-0001-9335-8762 Universidade do Estado do Rio de Janeiro, Brasil

E-mail: bruno.eduardo@mail.com

Aylla Monteiro Maconato

ORCID: https://orcid.org/0000-0001-7649-7840 Universidade do Estado do Rio de Janeiro, Brasil E-mail: aylla_maconato@hotmail.com

Daiane de Oliveira Campos da Veiga ORCID: https://orcid.org/0000-0001-8647-2997 Universidade do Estado do Rio de Janeiro, Brasil E-mail: anyrrcampos@hotmail.com

Sarah Kelley Ribeiro de Almeida ORCID: https://orcid.org/0000-0003-0563-1891 Universidade Federal do Rio de Janeiro, Brasil E-mail: sarahkelley@outlook.com.br

Thayná Victorio Costa Cavalcanti ORCID: https://orcid.org/0000-0001-8917-6018 Universidade do Estado do Rio de Janeiro, Brasil E-mail: enfermeirageronto@gmail.com

Rebecca Rodrigues de Barros

ORCID: https://orcid.org/0000-0003-4117-2696 Universidade Federal do Rio de Janeiro, Brasil E-mail: rebeccarbarros@ hotmail.com

Itaécio Felipe Silva

ORCID: https://orcid.org/0000-0003-4462-3886 Universidade Regional do Cariri, Brasil

E-mail: i_ta_ecio@hotmail.com

Isaac Neves de Lima

ORCID: https://orcid.org/0000-0002-2057-2597 Universidade Federal do Goiás, Brasil E-mail: issacneves@hotmail.com

Cristiane de Almeida Faria Abranches ORCID: https://orcid.org/0000-0002-3258-3656 Universidade do Estado do Rio de Janeiro, Brasil E-mail: c.isio@ hotmail.com

Michele Cristina de Oliveira

ORCID: https://orcid.org/0000-0002-4239-615X

Fundação de Apoio à Escola Técnica, Brasil E-mail: michelemco@gmail.com

\begin{abstract}
Resumo
Por meio desse material científico traçamos um estudo da velhice no que tange à sexualidade, e elencamos como descritores o corpo e o prazer, tão discutidos já no âmbito da filosofia e da psicanálise. Nesse sentido, nosso objetivo é realizar uma revisão de literatura da velhice e da sexualidade na pós-modernidade, por meio de um estudo que engloba o corpo e o prazer. Nosso pensamento é instituído por meio da seguinte assertiva: diferentemente do que rezam alguns preconceitos oriundos do senso comum, velhice não é sinônimo de incapacidade funcional, dependência ou ausência de vivências sociais e sexuais. Ancoramo-nos, portanto, na linha de pensamento que enfatiza a necessidade de resgatar o direito a uma vida sexual na velhice, uma vez que implica poder pensar o amor em suas formas de transformação libidinal. Por fim, ressaltamos que é preciso compreendê-lo em um sentido amplo, que passa pela ternura, pelos contatos físicos que erogenizam o corpo, como o olhar, a voz e o toque.
\end{abstract}

Palavras-chave: Velhice; Sexualidade; Corpo; Prazer. 


\begin{abstract}
Through this scientific material, we draw a study of old age with regard to sexuality, and we list body and pleasure as descriptors, so discussed in the scope of philosophy and psychoanalysis. In this sense, our goal is to conduct a review of the literature on old age and sexuality in postmodernity, through a study that encompasses the body and pleasure. Our thinking is instituted by means of the following statement: unlike what some common sense prejudices say, old age is not synonymous with functional disability, dependence or absence of social and sexual experiences. We are anchored, therefore, in the line of thought that emphasizes the need to rescue the right to a sexual life in old age, since it implies being able to think about love in its libidinal transformation forms. Finally, we emphasize that it is necessary to understand it in a wide range, which includes tenderness, physical contacts that erogenize the body, such as the look, the voice and the touch.
\end{abstract}

Keywords: Old age; Sexuality; Body; Pleasure.

\title{
Resumen
}

A través de este material científico, trazamos un estudio de la vejez en relación con la sexualidad, y enlistamos cuerpo y placer como descriptores, así discutidos en el ámbito de la filosofía y el psicoanálisis. En este sentido, nuestro objetivo es realizar una revisión de la literatura sobre la vejez y la sexualidad en la posmodernidad, a través de un estudio que abarque el cuerpo y el placer. Nuestro pensamiento se instituye mediante la siguiente afirmación: a diferencia de lo que dicen algunos prejuicios de sentido común, la vejez no es sinónimo de discapacidad funcional, dependencia o ausencia de experiencias sociales y sexuales. Estamos anclados, por tanto, en la línea de pensamiento que enfatiza la necesidad de rescatar el derecho a la vida sexual en la vejez, ya que implica poder pensar en el amor en sus formas de transformación libidinal. Finalmente, destacamos que es necesario entenderlo en un amplio rango, que incluye la ternura, los contactos físicos que erogenizan el cuerpo, como la mirada, la voz y el tacto.

Palabras clave: Vejez; Sexualidad; Cuerpo; Placer.

\section{Introdução}

O processo de envelhecimento humano é um tema que ganha palco nas discussões do ser humano desde a era helênica, evidenciando-se nos questionamentos advindos das culturas grega e romana. Desse modo, com intensidade após o século das luzes, vem despertando, ao longo dos anos, o interesse de pesquisadores das mais diversas áreas do conhecimento científico, dentre elas, a ciência psicológica (Cachioni; Falcão, 2009).

A Velhice: Realidade Incômoda, de Simone de Beauvoir (1970) ganha fundamental importância por ter se proposto a quebrar a "conspiração do silêncio" que circundava esse tema nos anos 70. Havia uma manipulação nos dados e um efeito de silenciamento da velhice, tida como um dos aspectos da Loucura (Foucault, 2002).

Em mesma proporção escalonada, a antropóloga Guita Grin Debert faz em seu livro, A Reinvenção da Velhice (1999)1, uma análise da transformação da velhice em um tema privilegiado. Nesse trabalho nós traçamos um estudo da velhice no que tange à sexualidade, e elencamos como descritores o corpo e o prazer, tão discutidos já no âmbito da filosofia e da psicanálise. Assegurados pela postura do Ministério da Saúde no Brasil, denota-se que "O envelhecimento é um processo sequencial, individual, cumulativo, irreversível, universal, não patológico, de deterioração de um organismo maduro, próprio a todos os membros de uma espécie" (Brasil, 2006).

Por um pequeno olhar que lançamos sobre as pesquisas percebemos um campo semântico em torno da sexualidade constituído por uma multiplicidade de elementos, desde fatores que advém da gerontologia a atos de cumplicidade, a intimidade, o ato sexual, dentre outros. Nesse sentido, nosso objetivo é realizar uma revisão de literatura da velhice e da sexualidade na pósmodernidade, por meio de um estudo que engloba o corpo e o prazer.

Nosso pensamento é instituído por meio da seguinte assertiva: diferentemente do que rezam alguns preconceitos oriundos do senso comum, velhice não é sinônimo de incapacidade funcional, dependência ou ausência de vivências sociais e

\footnotetext{
${ }^{1}$ A autora propõe que é necessário atentar para o duplo movimento que acompanha a transformação da velhice em uma preocupação social. Por um lado, percebe-se uma socialização progressiva da gestão da velhice. Isso é facilmente visualizado ao olhar um pouco para trás e constatar que até muito recente o cuidado com os idosos era uma preocupação restrita ao âmbito familiar ou, no máximo, relegado às associações filantrópicas. Atualmente, porém, o que se nota é um conjunto de intervenções e orientações, muitas vezes contraditórias, implementadas pelo aparelho Estatal e/ou pela sociedade civil. Até mesmo um campo de saber específico para a terceira idade foi criado: a gerontologia, especialistas no envelhecimento (Debert, 1999).
} 
sexuais. Mesmo na presença de perdas físicas, psicológicas e sociais decorrentes do processo de envelhecimento humano, é possível a vivência de uma velhice bem sucedida. De acordo com Cachioni e Falcão (2009).

Desse modo, após discutir inicialmente os aspectos teóricos da pesquisa, englobando elementos como a velhice, a sexualidade e o prazer, pincelamos uma breve discussão sobre algumas estratégias de governamentalidade para melhorar os corpos dos idosos.

\title{
2. Método
}

Por se tratar de uma revisão de literatura, a abordagem escolhida por nós foi a qualitativa, que se caracteriza pela qualificação dos dados coletados, a partir do momento em que discorremos sobre a problematização. Assim, "A pesquisa qualitativa não se preocupa com representatividade numérica, mas, sim, com o aprofundamento da compreensão de um grupo social, de uma organização etc." (Goldenberg, 1997, p. 34).

$\mathrm{Na}$ ótica deste pesquisador e analista, os pesquisadores que adotam a abordagem qualitativa atuam adversamente em relação ao pressuposto que defende um modelo único de pesquisa para todas as ciências, uma vez que as ciências sociais têm sua especificidade, o que pressupõe uma metodologia própria. "Assim, os pesquisadores qualitativos recusam o modelo positivista aplicado ao estudo da vida social, uma vez que o pesquisador não pode fazer julgamentos nem permitir que seus preconceitos e crenças contaminem a pesquisa" (Goldenberg, 1997, p. 34).

\begin{abstract}
A pesquisa qualitativa trabalha com o universo de significados, motivos, aspirações, crenças, valores e atitudes, o que corresponde a um espaço mais profundo das relações, dos processos e dos fenômenos que não podem ser reduzidos à operacionalização de variáveis. Aplicada inicialmente em estudos de Antropologia e Sociologia, como contraponto à pesquisa quantitativa dominante, tem alargado seu campo de atuação a áreas como a Psicologia e a Educação. A pesquisa qualitativa é criticada por seu empirismo, pela subjetividade e pelo envolvimento emocional do pesquisador (Minayo, 2001, p. 14).
\end{abstract}

Nesse sentido, tendo em vista que a base adotada é teórica, nossa pesquisa é bibliográfica, em que o analista se constitui de uma fonte segura como uma atividade em busca de significados e sentidos com outras atividades comunicativas. Assim segundo as autoras Marconi e Lakatos (1992),

A pesquisa bibliográfica é o levantamento de toda a bibliografia já publicada, em forma de livros, revistas, publicações avulsas e imprensa escrita. A sua finalidade é fazer com que o pesquisador entre em contato direto com todo o material escrito sobre um determinado assunto, auxiliando o cientista na análise de suas pesquisas ou na manipulação de suas informações. Ela pode ser considerada como o primeiro passo de toda a pesquisa científica (Marconi; Lakatos, 1992, p. 75)

No que concerne o processo de pesquisa, Gil (2007), refere-se à pesquisa podendo ser definida como "Um procedimento racional e sistemático que tem como objetivo proporcionar respostas aos problemas que são propostos... desde a formulação do problema até a apresentação e discussão dos resultados" (Gil, 2007, p. 17).

\section{Aspectos Teóricos: Velhice e Sexualidade, Corpo e Prazer}

Há muito o que se considerar, quando tratamos de uma temática tão densa quanto as questões de Ciência, raça e sexualidade no decorrer dos séculos a qual envolve não apenas uma perspectiva teórica de análise, mas entornos históricos que constituem aspectos psicofisiológicos do sujeito.

É possível perceber, por meio de estudos advindos da gerontologia, que cada vez mais as indústrias midiáticas tem produzido a ideia de que ao tempo cronológico necessita-se acrescentar vida ativa (Lima; Silva; Gahardoni, 2008), necessidade identificada como produto de esforços agenciados nos últimos anos, em grande medida, relacionados ao incremento da produção 
em ciência, tecnologia e práticas de atenção à saúde dos idosos (Lima; Silva; Gahardoni, 2008), Isso porque a velhice, enquanto condição vital e inevitável, constituinte das dimensões do ser, trata-se de um processo de mudanças universais, pautado geneticamente para a espécie e para cada indivíduo, que se traduz em diminuição da plasticidade, em aumento da vulnerabilidade (Neri, 2008; Silva, 2006) em acúmulo de perdas evolutivas e no aumento da probabilidade de morte.

Dados do Ministério da Saúde contemplam que a velhice se refere ao ato ou efeito de envelhecer, que significa ficar velho (Brasil, 2006); parecer velho; durar muito tempo, permanecer, tornar-se desusado ou inútil (Neri, 2008; Silva, 2006). Quando se pensa, então, na sexualidade, ou seja, nas diversas produções e estigmas acerca da prática do sexo, as considerações, em grande maioria, enxergam que os idosos são inférteis, que já não podem ter uma vida sexual saudável como, por exemplo, os jovens.

Tal visão associa imediatamente à questão do prazer, resultando em uma fórmula abstrata para a terceira idade: os idosos não fazem mais sexo, e quando o fazem, não sentem prazer. A concepção pioneira de Freud, quando afirma que o prazer é um objetivo da sexualidade humana, desassocia as vivências sexuais do significado exclusivo da reprodução².

É preciso considerar que, de acordo com Pascual (2002), existe, em nossa sociedade, um conceito de velhice deteriorado e negativo, especialmente no âmbito sexual.

O processo de envelhecimento não conduz a uma fase assexuada, mas tão somente a outra etapa no processo da sexualidade humana, a qual deve ser merecidamente vivenciada e apreciada (Fávero \& Barbosa, 2011). As vivências sexuais, independentemente da idade, proporcionam ao casal a possibilidade de se realizar pessoalmente, refletem a intimidade e a cumplicidade e enriquecem as relações humanas. Para os idosos, a sexualidade é fisiologicamente possível, emocional e afetivamente enriquecedora, porquanto fortalece a importância do carinho, do apego, a comunicação, o companheirismo e o cuidado mútuo (Urquiza, et al., 2008).

Torna-se imprescindível que compreendamos que, de acordo com Pires, o tempo não dessexualiza a pessoa idosa, uma vez que a sexualidade está presente em todas as fases da vida, percorre "um caminho de faz e refaz, um caminho instável, em constante processo de transformação, assim como as pessoas, pois é parte indissociável delas" (Pires, 2006, p.2). Essa concepção nos faz repensar os estigmas produzidos ao relacionar diretamente a pessoa idosa a dificuldades no sexo ou à ausência do prazer, convocando os princípios da impotência sexual.

$\mathrm{Na}$ fase da terceira idade, ou seja, na idade ativa dos idosos, a sexualidade varia tanto quanto os demais comportamentos, mas isso não implica necessariamente uma redução drástica da resposta sexual (Pascual, 2002), já que ela depende fundamentalmente da atitude que cada pessoa adota diante da vida. Ocorre de maneira extremamente individual e não se processa do mesmo modo em todas as épocas, nem sequer da mesma forma em todos os indivíduos (Pascual, 2002).

Concordamos com Vieira, Miranda e Coutinho sobre o fato de que a sexualidade na velhice, devido a sua complexidade, necessita ser vista sob um novo olhar que não a limite apenas aos seus aspectos biológicos, uma vez que se trata de uma construção social (Vieira, Miranda, Coutinho, 2012). É preciso atentar para as necessidades impostas pela velhice e para a busca de possibilidades de assistência integral ao segmento idoso (Brasil, 2006). Dessa forma, conhecer as representações sociais da

\footnotetext{
${ }^{2}$ Sua tese foi confirmada com a recente emergência do conceito de saúde sexual e com a dissociação progressiva do conceito de reprodução, o que coloca em evidência a autonomização da vida sexual e sua importância para a realização e o bem-estar dos indivíduos durante toda a vida (Sousa, 2008).

Outros avanços no estudo das vivências sexuais foram realizados por Masters e Johnson (1988), cujas pesquisas são consideradas um marco no estudo da sexualidade. Esses autores foram pioneiros no estudo da fisiologia da resposta sexual e acreditavam que, para entender a complexidade da sexualidade humana, era preciso conhecer a anatomia e a fisiologia sexual e ter dados psicológicos e sociológicos. Segundo os referidos autores, existem mitos culturais acerca dessa temática, nos grupos constituídos por crianças, idosos e portadores de alguma patologia, os quais são vistos como seres assexuados.

Socialmente, tem-se considerado a pessoa idosa como assexuada, desprovida de desejos e de vida sexual, como se os anos lhe trouxessem uma inapetência neste aspecto vital do desenvolvimento humano (Gonzalez \& Brenes, 2007). Entretanto, a literatura atual tem demonstrado não existirem razões fisiológicas que impeçam as pessoas idosas, em condições satisfatórias de saúde, de apresentarem uma vida sexual ativa.
} 
sexualidade na velhice significa compreender o significado deste fenômeno para a população idosa, investigando a presença dos mitos e preconceitos que permeiam este processo e que norteiam o comportamento dos idosos no que diz respeito às suas vivências sexuais. Diante do exposto, a presente pesquisa foi realizada com o objetivo de apreender as representações sociais da sexualidade na velhice elaboradas pelos idosos (Vieira, Miranda, Coutinho, 2012).

"Na medida em que a sociedade, de uma forma geral, provê recursos financeiros e intelectuais para a existência de uma terceira idade sã, o idoso decadente é visto como relapso e culpado de sua própria decrepitude". (Portal do Envelhecimento, 2019). Um fato que nos chama a atenção e enfatiza que:

[...] a visibilidade conquistada por experiências inovadoras e bem sucedidas decorrentes e consequentes da reprivatização do envelhecimento, fecha o espaço e os olhos da sociedade para as situações de abandono e maus-tratos (Portal do Envelhecimento, 2019) ${ }^{3}$.

Segundo Vala e Monteiro (2004), as representações sociais apresentam uma dimensão funcional e prática, que tem por consequência ser evidenciada na organização dos comportamentos, das atividades comunicativas, na argumentação e na explicação cotidianas e na diferenciação dos grupos sociais. A elaboração e o funcionamento de uma representação podem ser compreendidos por meio dos processos de objetivação e ancoragem.

\section{Estratégias de Governamentalidade para Melhorar os Corpos dos Idosos ${ }^{4}$}

O governo do Brasil, por meio da Organização Pan-americana da Saúde (OPAS) e a Organização Mundial da Saúde), lançou, em 2018, a Estratégia Brasil Amigo da Pessoa Idosa. A elaboração da iniciativa, que tem por objetivo alcançar o envelhecimento ativo, saudável, cidadão e sustentável para todos os brasileiros, contou com a colaboração da Organização PanAmericana da Saúde/Organização Mundial da Saúde (OPAS/OMS, 2018). Isso nos faz pensar que em decorrência do crescimento da população idosa nas últimas décadas, é preciso que estas tenham uma vida mais saudável, inclusive para que possam ser mais ativas no mercado de trabalho.

Na Estratégia, o Brasil atende às recomendações da OMS para avaliação e desenvolvimento dos Planos de Ação voltados à adaptação das cidades às necessidades dos idosos (OPAS/OMS, 2018). Ao todo oito domínios da vida urbana podem influenciar na saúde e na qualidade de vida dessa população:

- Espaços ao ar livre e edifícios;

- Transportes;

- Habitação;

- Participação social;

- Respeito e integração social;

- Participação cívica e emprego;

• Comunicação e informação;

\footnotetext{
${ }^{3}$ Uma visão de cunho psicológico pode ser evidenciada em Memória e Sociedade de Ecléa Bosi (1979). A tese da autora, segundo a introdução do livro feita por Marilena Chaú, é de que o velho não tem armas, nós é que temos que lutar por ele. A sociedade capitalista desarma o velho, mobilizando mecan ismos pelos quais oprime a velhice, destrói os apoios da memória e substitui a lembrança pela história oficial celebrativa. Para B osi (1979), oprime-se o idoso por intermédio de mecanismos institucionais: a burocracia da aposentadoria e dos asilos; psicológicos: a tutelagem, a recusa do diálogo, o b animento e a discriminação; técnicos: as próteses e a precariedade existencial daqueles que não podem adquiri-las; e científicos: as "pesquisas" que demonstram a incapacidade e a incompetência social do velho (Portal do Envelhecimento, 2019). Disponível em: https://www.portaldoenvelhecimento.com.br/estudos-sobre-a-velhice/ Acesso em: 25.08.2020 O trabalho de Bosi, longe de se encerrar na constatação da opressão a que está submetida a memória dos velhos, procura encontrar a gênese dessa opressão. Segundo ela, a degradação senil começa prematuramente com a degradação da pessoa que trabalha. A nossa sociedade pragmática, para ela, não desvaloriza somente o operário, mas todo trabalhador. Bosi conclui que para reparar a destruição sistemática que os indivíduos sof rem na sociedade da competição e do lucro e para que o indivíduo, na velhice, permaneça um indivíduo, seria necessário que ele sempre tivesse sido tratado como u m indivíduo. Para ela, a noção que se tem de velhice decorre mais da luta de classes do que do conflito de gerações (Portal do Envelhecimento, 2019). Disponível em: https://www.portaldoenvelhecimento.com.br/estudos-sobre-a-velhice/ Acesso em: 25.08.2020.

${ }^{4}$ Disponível em: https://www.paho.org/bra/index.php?option=com_content\&view=article\&id=5625:brasil-lanca-estrategia-para-melhorar-vida-de-idosos-combase-em-recomendacoes-da-oms\&Itemid=820 Acesso em: 24.07.2020
} 
- Apoio da comunidade e serviços de saúde (OPAS/OMS, 2018).

Além dessas, mais uma dimensão foi oferecida para que os municípios brasileiros tenham flexibilidade ao inserir ações que considerem não contempladas nas oito dimensões: "Escolhas Locais"5.

Acerca do envelhecimento ativo, o número de pessoas com idade igual ou superior a 60 anos vai mais que dobrar no mundo em 2050, passando de 900 milhões em 2015 para cerca de 2 bilhões. Por isso, a Organização Pan-Americana da Saúde/Organização Mundial da Saúde (OPAS/OMS, 2018) acredita ser importante que os idosos de hoje e os do futuro possam envelhecer de maneira saudável e ativa. Ou seja, que a idade avançada não impeça as pessoas de ser e fazer o que querem ou valorizam.

Em retorno a Pascual (2002) sobre a existência de conceitos deteriorados e deficientes sobre a velhice na pósmodernidade, o que acaba comprometendo o entendimento que se tem sobre o prazer, atentamos ao fato de que ainda não há o apoio por parte de alguns profissionais de saúde, ao mesmo tempo em que os familiares põem obstáculos para impedir que seus idosos continuem sendo sexualmente ativos. Além disso, os meios de comunicação proporcionam uma visão pouco atrativa do processo de envelhecimento e, consequentemente, da pessoa idosa.

Tal fato é extremamente prejudicial aos idosos, haja vista que a sexualidade é um componente fundamental da qualidade de vida, essencial para manter as relações interpessoais saudáveis, o autoconceito e um senso de integridade. Está ligada ao senso de autoestima e, se negada, pode ter efeitos deletérios não só sobre a sexualidade em si, mas também em uma autoimagem, relações sociais e saúde mental (Bauer, Mcauliffe e Nay, 2007). Nessas dialogações, quando se fala de sexualidade e prazer na velhice, é preciso considerar uma série de problemáticas existentes que pairam em torno da terceira idade, conforme divulgado por pesquisas da OMS e do Ministério da Saúde no Brasil.

Concordamos, por fim, com Santos e Carlos (2003), que enfatizam que resgatar o direito a uma vida sexual na velhice implica poder pensar o amor em suas formas de transformação libidinal. Significa compreendê-lo em um sentido amplo, que passa pela ternura, pelos contatos físicos que erogenizam o corpo, como o olhar, o toque, a voz, redescobrindo as primeiras formas de amor do ser humano (Santos; Carlos, 2003).

\section{Considerações Finais}

O discurso dos idosos participantes ficou focalizado em quatro eixos temáticos: os elementos constitutivos da sexualidade, a sua importância e sua repercussão na vida do idoso, assim como a apreciação da sociedade acerca da sexualidade da pessoa idosa. $\mathrm{Na}$ apreensão das representações sociais da sexualidade destacaram-se ancoragens em aspectos vitais, relação corpo/sexo, elementos psicoafetivos e sociais. As ambivalências concentram-se na avaliação dos participantes quanto à importância das vivências sexuais e evoluímos na percepção da sociedade acerca da sexualidade dos idosos (Carlos, 2003).

$\mathrm{O}$ envelhecimento populacional representa um fenômeno mundial que vem demandando desafios constantes aos profissionais de diversas áreas do conhecimento científico. Os indicadores, sejam de ordem biológica, psicológica ou social, apontam para a necessidade de redefinição de conceitos e valores, no sentido de garantir um envelhecimento ativo e socialmente inserido (Brasil, 2006).

Acreditamos que cumprimos que cumprimos com o rito exigido de composição de um artigo, desde as informações coletadas até a correlação semântica dos elementos. Compreender o processo de envelhecimento considerando apenas os seus

\footnotetext{
${ }^{5}$ Entre as diretrizes da estratégia estão a observância das dimensões de avaliação de comunidades e cidades conforme metodologia da OMS adaptada às particularidades da realidade brasileira; a execução municipal orientada pelo Governo Federal do Brasil e pela OPAS e avaliada pelo Estado; e a participação dos Conselhos Municipais dos Direitos da Pessoa Idosa, inclusive em validações.

A iniciativa terá como prioridades o combate à violência e ao abuso financeiro, psicológico ou físico contra o idoso, bem como a adaptação de residências para essa população, atividades formativas, medidas de prevenção de quedas e cuidados em saúde; entre outras.
} 
aspectos negativos impossibilita a percepção de fatores importantes que são vivenciados apenas nessa etapa do desenvolvimento, como a experiência (Brasil, 2006).

Entretanto, em palavras não findas, esperamos que este trabalho possa inspirar e/ou impulsionar novas pesquisas, que se desdobrem nos intercaminhos sobre a velhice e a sexualidade. Que este trajeto por nós traçado aguce o senso crítico de novos leitores, cientistas e profissionais que lidam com o corpo velho e o prazer, e que os convoquem novas discussões pertinentes a temática abordada no estudo.

\section{Referências}

Bauer, M., Mcauliffe, L., \& Nay, R. (2007). Sexuality, health care and the older person: an overview of the literature. International Journal of Older People Nursing, 2 (1), 63-68, Brasil.

Brasil. Ministério da Saúde, Secretaria de Atenção à Saúde, Departamento de Atenção Básica. (2006). Envelhecimento e saúde da pessoa idosa (Cadernos de Atenção Básica - n. ${ }^{\circ}$ 19, Série A: envelhecimento e saúde da pessoa idosa). Brasília, DF.

Beauvoir, S. (1970). A Velhice: Realidade Incômoda. $2^{\text {a }}$ Edição. Editora: Difusão Europeia.

Cachioni, M., \& Falcão, D. V. S. (2009). Velhice e Educação: possibilidades e benefícios para a qualidade de vida. Em D.V.S. Falcão, \& L.F. Araújo (Eds.), Psicologia do envelhecimento: relações sociais, bem-estar subjetivo e atuação profissional em contextos diferenciados. Campinas: Alínea, 2009, pp. $175-194$.

Debert, G.G. (1999). A reinvenção da velhice. EDUSP Editora.

Fávero, M. F., \& Barbosa, S. C. S. (2011). Sexualidade na velhice: os conhecimentos e as atitudes dos profissionais de saúde. Terapia Sexual, 14(2), 11-39.

Foucault, M. História da sexualidade 1: a vontade de saber.

Freud, S. (1996) Três ensaios sobre a teoria da sexualidade. Imago. (Trabalho original publicado em 1905).

Gil, A. C. (2007). Métodos e técnicas de pesquisa social. (5a ed.), Atlas.

Goldenberg, M. (1997). A arte de pesquisar: como fazer pesquisa qualitativa em ciências sociais. (4a ed.), Record.

Lima, A. M. M., silva, H. S., \& Galhardoni, S. (2008). Successful aging: paths for a construct and new frontiers. Interface - Comunicação, Saúde, Educação, 12(27), 795-807. Brasil.

Marconi, M. A., \& Lakatos, E. M. (1992). Metodologia do trabalho científico. Atlas. (4a ed.).

Minayo, M. C. S. (2001). Pesquisa Social: teoria, método e criatividade. (18a ed.), Vozes.

Neri, A. L. (2008). Palavras-chave em gerontologia. Ed. Alínea. Campinas, SP.

Neri, A. L. (2002). Teorias psicológicas do envelhecimento. In. Freitas, E. V. (Ed.), Tratado de geriatria e gerontologia (pp. 32-45). Guanabara Koogan, Rio de Janeiro, RJ.

OPAS. Organização Pan-Americana da Saúde/Organização Mundial Da Saúde. (2018).

PAHO Brasil. (2018). Brasil lança estratégia para melhorar vida de idosos com base em recomendações da OMS.

Pascual, C. P. (2002). A sexualidade do idoso vista com novo olhar. Loyola.

Pires, R. C. C. A. (2006). Sexualidade feminina, envelhecimento e educação: algumas aproximações necessárias. Revista UDESC, 7(1), 1-7.

Portal do Envelhecimento. (2019). Brasil.

Silva, R. M. O. (2006). Sexualidade no idoso. In, Hargreaves, L. H. H. (Ed.), Geriatria (pp. 141-148). Brasília, DF: SEEP.

Urquiza, A., Tumala, D., Arnold-Cathalifaud, M., Ojeda, A., \& Vogel, N. (2008). Sexualidad em la tercera edad: la imagen de los jóvenes universitários. Ponto e Vírgula, 4, 358-374. Chile.

Vieira, K. F. L., Miranda, R. S., \& Coutinho, M. P. L. (2012). Sexualidade na velhice: um estudo de representações sociais. Psicologia e Saber Social, 1(1), $120-128$.

Vieira, K. F. L. (2012). Sexualidade e qualidade de vida do Idoso: desafios contemporâneos e repercussões sociais. (Tese de Doutorado), Universidade Federal da Paraíba, João Pessoa, PB. 